\title{
OPTION PRICING IN BILATERAL GAMMA STOCK MODELS
}

\author{
UWE KÜCHLER AND STEFAN TAPPE
}

\begin{abstract}
In the framework of bilateral Gamma stock models we seek for adequate option pricing measures, which have an economic interpretation and allow numerical calculations of option prices. Our investigations encompass Esscher transforms, minimal entropy martingale measures, $p$-optimal martingale measures, bilateral Esscher transforms and the minimal martingale measure. We illustrate our theory by a numerical example.
\end{abstract}

Key Words: Bilateral Gamma stock model, bilateral Esscher transform, minimal martingale measure, option pricing.

91G20, 60G51

\section{INTRODUCTION}

An issue in continuous time finance is to find realistic and analytically tractable models for price evolutions of risky financial assets. In this text, we consider exponential Lévy models

$$
\left\{\begin{aligned}
S_{t} & =S_{0} e^{X_{t}} \\
B_{t} & =e^{r t}
\end{aligned}\right.
$$

consisting of two financial assets $(S, B)$, one dividend paying stock $S$ with dividend rate $q \geq 0$, where $X$ denotes a Lévy process and one risk free asset $B$, the bank account with fixed interest rate $r \geq 0$. Note that the classical Black-Scholes model is a special case by choosing $X_{t}=\sigma W_{t}+\left(\mu-\frac{\sigma^{2}}{2}\right) t$, where $W$ is a Wiener process, $\mu \in \mathbb{R}$ denotes the drift and $\sigma>0$ the volatility.

Although the Black-Scholes model is a standard model in financial mathematics, it is well-known that it only provides a poor fit to observed market data, because typically the empirical densities possess heavier tails and much higher located modes than fitted normal distributions.

Several authors therefore suggested more sophisticated Lévy processes with a jump component. We mention the Variance Gamma processes [30, 31, hyperbolic processes [8], normal inverse Gaussian processes [1], generalized hyperbolic processes [9, 10, 34, CGMY processes [3] and Meixner processes [39]. A survey about Lévy processes used for applications to finance can for instance be found in 6 , Chap. 4] or [38, Chap. 5.3].

Recently, the class of bilateral Gamma processes, which we shall henceforth deal with in this text, was proposed in [27]. We also mention the related article [28, where the shapes of their densities are investigated.

Now, let $(S, B)$ be an exponential Lévy model of the type (1.1). In practice, we often have to deal with adequate pricing of European options $\Phi\left(S_{T}\right)$, where $T>0$ denotes the time of maturity and $\Phi: \mathbb{R} \rightarrow \mathbb{R}$ the payoff function. For example, the payoff profile of a European call option is $\Phi(x)=(x-K)^{+}$.

The option price is given by $e^{-r T} \mathbb{E}_{\mathbb{Q}}\left[\Phi\left(S_{T}\right)\right]$, where $\mathbb{Q} \sim \mathbb{P}$ is a local martingale measure, i.e., a probability measure, which is equivalent to the objective probability measure $\mathbb{P}$, such that the discounted stock price process

$$
\tilde{S}_{t}:=e^{-(r-q) t} S_{t}=S_{0} e^{X_{t}-(r-q) t}, \quad t \geq 0
$$


is a local $\mathbb{Q}$-martingale, where $q$ denotes the dividend rate. Note that $\left(\tilde{S}_{t}\right)_{t \geq 0}$ also has the interpretation as the discounted value process of an investor who is endowed with one stock and reinvests all dividend payments $-q S_{t} d t$ per share - in new shares of the same stock. Indeed, integration by parts shows that

$$
\begin{aligned}
e^{-r t} S_{t}=\tilde{S}_{t} e^{-q t} & =S_{0}+\int_{0}^{t} e^{-q s} d \tilde{S}_{s}-\int_{0}^{t} \tilde{S}_{s} q e^{-q s} d s \\
& =S_{0}+\int_{0}^{t} e^{-q s} d \tilde{S}_{s}-\int_{0}^{t} e^{-r s} q S_{s} d s, \quad t \geq 0
\end{aligned}
$$

and therefore the discounted price process $\left(\tilde{S}_{t}\right)_{t \geq 0}$ is a local $\mathbb{Q}$-martingale if and only if the process

$$
e^{-r t} S_{t}+\int_{0}^{t} e^{-r s} q S_{s} d s, \quad t \geq 0
$$

is a local $\mathbb{Q}$-martingale.

Typically, exponential Lévy models (1.1) are free of arbitrage, but, unlike the classical Black-Scholes model, not complete, that is, there exist several martingale measures $\mathbb{Q} \sim \mathbb{P}$. In particular, if not enough market data for calibration are available, we are therefore faced with problem, which martingale measure we should choose.

This text is devoted to the existence of suitable pricing measures $\mathbb{Q} \sim \mathbb{P}$ for bilateral Gamma stock models. We will in particular focus on the following two criteria:

- Under $\mathbb{Q}$, the process $X$ should again be a bilateral Gamma process, because this allows, by virtue of the simple characteristic function (3.1) below, numerical calculations of option prices by using the method of Fourier transformation.

- The measure $\mathbb{Q}$ should have a reasonable economic interpretation.

The basic tool for all subsequent calculations in this paper is the cumulant generating function (3.4) below of the bilateral Gamma distribution. There exists a more general class of infinitely divisible distributions, the so-called tempered stable distributions (see [6, Sec. 4.5]), which also include the CGMY distributions. They have a comparatively simple cumulant generating function, too, and thus analogous results for essential parts of the present paper can also be proven for them. However, their cumulant generating functions considerably differ from that of bilateral Gamma distributions, in particular they have finite values at the boundary of their domains, which is not true for cumulant generating functions of bilateral Gamma distributions.

In order to avoid a cumbersome presentation of this article, we shall provide the concrete results for tempered stable processes in a subsequent paper, in which we also investigate further properties of this family of distributions. Nevertheless, in this text, we shall always indicate the corresponding results for tempered stable distributions - without going into detail - and compare it with the results derived for bilateral Gamma distributions.

One practical advantage of bilateral Gamma distributions is the explicit form of the density, which we do not have for general tempered stable distributions. This allows to apply maximum likelihood estimators in order to determine the parameters from observations of a stock.

The remainder of this text is organized as follows. In Section 2 we provide the required results from stochastic calculus. In Section 3 we review bilateral Gamma processes and introduce bilateral Gamma stock models. Sections 47 are devoted to several approaches on choosing suitable martingale measures for option pricing 
in bilateral Gamma stock models. We conclude with a numerical illustration in Section 8 .

\section{Prerequisites from stochastic Calculus}

In this section, we collect the results from stochastic calculus, which we will require in the sequel. Throughout this text, let $\left(\Omega, \mathcal{F},\left(\mathcal{F}_{t}\right)_{t \geq 0}, \mathbb{P}\right)$ be a filtered probability space satisfying the usual conditions.

2.1. Lemma. 19, Thm. I.4.61] Let $X$ be a real-valued semimartingale. There exists a unique (up to indistinguishability) solution $Z$ for the equation

$$
Z_{t}=1+\int_{0}^{t} Z_{s-} d X_{s}, \quad t \geq 0 .
$$

2.2. Definition. Let $X$ be a real-valued semimartingale. We call the unique solution $Z$ for 2.1 the stochastic exponential or Doléans-Dade exponential of $X$ and write $\mathcal{E}(X):=Z$.

2.3. Lemma. 22, Lemma 2.2] Let $Z$ be a semimartingale such that $Z, Z$ - are $\mathbb{R} \backslash\{0\}$-valued. There exists a unique (up to indistinguishability) semimartingale $X$ such that $X_{0}=0$ and $Z=Z_{0} \mathcal{E}(X)$. It is given by

$$
X_{t}=\int_{0}^{t} \frac{1}{Z_{s-}} d Z_{s}, \quad t \geq 0 .
$$

2.4. Definition. Let $Z$ be a semimartingale such that $Z, Z_{-}$are $\mathbb{R} \backslash\{0\}$-valued. We call the unique process $X$ from Lemma 2.3 the stochastic logarithm of $Z$ and write $\mathcal{L}(Z):=X$.

2.5. Lemma. Let $X$ be a real-valued Lévy process. The following statements are equivalent:

(1) $X$ is a local martingale;

(2) $X$ is a martingale;

(3) $\mathbb{E}\left[X_{1}\right]=0$.

Proof. For $(1) \Leftrightarrow(2)$ see $\left[42\right.$. Noting that $\mathbb{E}\left[X_{t}\right]=t \mathbb{E}\left[X_{1}\right]$ for all $t \geq 0$, the equivalence $(2) \Leftrightarrow(3)$ follows from [6, Prop. 3.17].

2.6. Lemma. Let $X$ be a real-valued Lévy process. The following statements are equivalent:

(1) $e^{X}$ is a local martingale;

(2) $e^{X}$ is a martingale;

(3) $\mathbb{E}\left[e^{X_{1}}\right]=1$.

Proof. The equivalence $(1) \Leftrightarrow(2)$ follows from [21, Lemma 4.4.3]. Noting that $\mathbb{E}\left[e^{X_{t}}\right]=\left(\mathbb{E}\left[e^{X_{1}}\right]\right)^{t}$ for all $t \geq 0$, the equivalence $(2) \Leftrightarrow(3)$ follows from 6 , Prop. $3.17]$.

\section{Stock price models Driven by bilateral Gamma processes}

In this section, we shall introduce bilateral Gamma stock models. For this purpose, we first review the family of bilateral Gamma processes. For details and more information, we refer to 27 .

A bilateral Gamma distribution with parameters $\alpha^{+}, \lambda^{+}, \alpha^{-}, \lambda^{-}>0$ is defined as the distribution of $Y-Z$, where $Y$ and $Z$ are independent, $Y \sim \Gamma\left(\alpha^{+}, \lambda^{+}\right)$and 
$Z \sim \Gamma\left(\alpha^{-}, \lambda^{-}\right)$. For $\alpha, \lambda>0$ we denote by $\Gamma(\alpha, \lambda)$ a Gamma distribution, i.e. the absolutely continuous probability distribution with density

$$
f(x)=\frac{\lambda^{\alpha}}{\Gamma(\alpha)} x^{\alpha-1} e^{-\lambda x} \mathbb{1}_{(0, \infty)}(x), \quad x \in \mathbb{R} .
$$

The characteristic function of a bilateral Gamma distribution is given by

$$
\phi(z)=\left(\frac{\lambda^{+}}{\lambda^{+}-i z}\right)^{\alpha^{+}}\left(\frac{\lambda^{-}}{\lambda^{-}+i z}\right)^{\alpha^{-}}, \quad z \in \mathbb{R}
$$

where the powers stem from the main branch of the complex logarithm.

Thus, any bilateral Gamma distribution is infinitely divisible, which allows us to define its associated Lévy process $\left(X_{t}\right)_{t \geq 0}$, which we call a bilateral Gamma process. We write $X=\left(X_{t}\right)_{t>0} \sim \Gamma\left(\alpha^{+}, \lambda^{+} ; \alpha^{-}, \lambda^{-}\right)$if $X_{1}$ has a bilateral Gamma distribution with parameters $\alpha^{+}, \lambda^{+}, \alpha^{-}, \lambda^{-}>0$. All increments of $X$ have a bilateral Gamma distribution, more precisely

$$
X_{t}-X_{s} \sim \Gamma\left(\alpha^{+}(t-s), \lambda^{+} ; \alpha^{-}(t-s), \lambda^{-}\right) \text {for } 0 \leq s<t .
$$

The characteristic triplet with respect to the truncation function $h \equiv 0$ is given by $(0,0, F)$, where $F$ denotes the Lévy measure

$$
F(d x)=\left(\frac{\alpha^{+}}{x} e^{-\lambda^{+} x} \mathbb{1}_{(0, \infty)}(x)+\frac{\alpha^{-}}{|x|} e^{-\lambda^{-}|x|} \mathbb{1}_{(-\infty, 0)}(x)\right) d x .
$$

The cumulant generating function $\Psi(z)=\ln \mathbb{E}\left[e^{z X_{1}}\right]$ exists on $\left(-\lambda^{-}, \lambda^{+}\right)$and is given by

$$
\Psi(z)=\alpha^{+} \ln \left(\frac{\lambda^{+}}{\lambda^{+}-z}\right)+\alpha^{-} \ln \left(\frac{\lambda^{-}}{\lambda^{-}+z}\right), \quad z \in\left(-\lambda^{-}, \lambda^{+}\right) .
$$

We can write $X=X^{+}-X^{-}$as the difference of two independent standard Gamma processes, where $X^{+} \sim \Gamma\left(\alpha^{+}, \lambda^{+}\right)$and $X^{-} \sim \Gamma\left(\alpha^{-}, \lambda^{-}\right)$. The corresponding cumulant generating functions $\Psi^{+}(z)=\ln \mathbb{E}\left[e^{z X_{1}^{+}}\right]$and $\Psi^{-}(z)=\ln \mathbb{E}\left[e^{z X_{1}^{-}}\right]$are given by

$$
\begin{aligned}
& \Psi^{+}(z)=\alpha^{+} \ln \left(\frac{\lambda^{+}}{\lambda^{+}-z}\right), \quad z \in\left(-\infty, \lambda^{+}\right) \\
& \Psi^{-}(z)=\alpha^{-} \ln \left(\frac{\lambda^{-}}{\lambda^{-}-z}\right), \quad z \in\left(-\infty, \lambda^{-}\right) .
\end{aligned}
$$

Note that $\Psi(z)=\Psi^{+}(z)+\Psi^{-}(-z)$ for $z \in\left(-\lambda^{-}, \lambda^{+}\right)$.

A bilateral Gamma stock model is an exponential Lévy model of the type (1.1) with $X$ being a bilateral Gamma process. In what follows, we assume that $r \geq q \geq 0$, that is, the dividend rate $q$ of the stock cannot exceed the interest rate $r$ of the bank account and none of them is negative.

3.1. Lemma. Let $X \sim \Gamma\left(\alpha^{+}, \lambda^{+} ; \alpha^{-}, \lambda^{-}\right)$under $\mathbb{P}$.

(1) If $\lambda^{+}>1$, then $\mathbb{P}$ is a martingale measure for $\tilde{S}$ if and only if

$$
\left(\frac{\lambda^{+}}{\lambda^{+}-1}\right)^{\alpha^{+}}\left(\frac{\lambda^{-}}{\lambda^{-}+1}\right)^{\alpha^{-}}=e^{r-q}
$$

(2) If $\lambda^{+} \leq 1$, then $\mathbb{P}$ is never a martingale measure for $\tilde{S}$.

Proof. If $\lambda^{+}>1$, we have $\mathbb{E}\left[e^{X_{1}}\right]<\infty$. By Lemma 2.6 the discounted price process $\tilde{S}$ in $\left[1.2\right.$ is a local martingale if and only if $\mathbb{E}\left[e^{X_{1}-(r-q)}\right]=1$, which is the case if and only if 3.7 holds.

In the case $\lambda^{+} \leq 1$ we have $\mathbb{E}\left[e^{X_{1}}\right]=\infty$. Lemma 2.6 implies that $\tilde{S}$ cannot be a local martingale. 


\section{Existence of Esscher martingale measures in Bilateral Gamma STOCK MODELS}

For option pricing in bilateral Gamma stock models of the type 1.1) we have to find a martingale measure. One method is to use the so-called Esscher transform, which was pioneered in [14. We recall the definition in the context of bilateral Gamma processes.

4.1. Definition. Let $X \sim \Gamma\left(\alpha^{+}, \lambda^{+} ; \alpha^{-}, \lambda^{-}\right)$under $\mathbb{P}$ and let $\Theta \in\left(-\lambda^{-}, \lambda^{+}\right)$be arbitrary. The Esscher transform $\mathbb{P}^{\Theta} \stackrel{\text { loc }}{\sim} \mathbb{P}$ is defined as the locally equivalent probability measure with likelihood process

$$
\Lambda_{t}\left(\mathbb{P}^{\Theta}, \mathbb{P}\right):=\left.\frac{d \mathbb{P}^{\Theta}}{d \mathbb{P}}\right|_{\mathcal{F}_{t}}=e^{\Theta X_{t}-\Psi(\Theta) t}, \quad t \geq 0
$$

where $\Psi$ denotes the cumulant generating function given by 3.4).

4.2. Lemma. Let $X \sim \Gamma\left(\alpha^{+}, \lambda^{+} ; \alpha^{-}, \lambda^{-}\right)$under $\mathbb{P}$ and let $\Theta \in\left(-\lambda^{-}, \lambda^{+}\right)$be arbitrary. Then we have $X \sim \Gamma\left(\alpha^{+}, \lambda^{+}-\Theta ; \alpha^{-}, \lambda^{-}+\Theta\right)$ under $\mathbb{P}^{\Theta}$.

Proof. This follows from Proposition 2.1.3 and Example 2.1.4 in [26].

The upcoming result characterizes all bilateral Gamma stock models, for which martingale measures given by an Esscher transform exist.

4.3. Theorem. Let $X \sim \Gamma\left(\alpha^{+}, \lambda^{+} ; \alpha^{-}, \lambda^{-}\right)$under $\mathbb{P}$. Then there exists $\Theta \in\left(-\lambda^{-}, \lambda^{+}\right)$ such that $\mathbb{P}^{\Theta}$ is a martingale measure if and only if

$$
\lambda^{+}+\lambda^{-}>1
$$

If (4.1) is satisfied, $\Theta$ is unique, belongs to the interval $\left(-\lambda^{-}, \lambda^{+}-1\right)$, and it is the unique solution of the equation

$$
\left(\frac{\lambda^{+}-\Theta}{\lambda^{+}-\Theta-1}\right)^{\alpha^{+}}\left(\frac{\lambda^{-}+\Theta}{\lambda^{-}+\Theta+1}\right)^{\alpha^{-}}=e^{r-q}, \quad \Theta \in\left(-\lambda^{-}, \lambda^{+}-1\right) .
$$

Moreover, we have $X \sim \Gamma\left(\alpha^{+}, \lambda^{+}-\Theta ; \alpha^{-}, \lambda^{-}+\Theta\right)$ under $\mathbb{P}^{\Theta}$.

Proof. Let $\Theta \in\left(-\lambda^{-}, \lambda^{+}\right)$be arbitrary. In view of Lemma 4.2 and Lemma 3.1 the probability measure $\mathbb{P}^{\Theta}$ is a martingale measure if and only if $\lambda^{+}-\Theta>1$, i.e. $\Theta \in\left(-\lambda^{-}, \lambda^{+}-1\right)$, and 4.2 is fulfilled. Note that $\left(-\lambda^{-}, \lambda^{+}-1\right) \neq \emptyset$ if and only if 4.1) is satisfied.

Provided 4.1, equation 4.2 is satisfied if and only if

$$
f(\Theta)=r-q
$$

where $f:\left(-\lambda^{-}, \lambda^{+}-1\right) \rightarrow \mathbb{R}$ is defined as $f(\Theta):=f^{+}(\Theta)+f^{-}(\Theta)$ with

$$
\begin{aligned}
& f^{+}(\Theta):=\alpha^{+}\left(\ln \left(\lambda^{+}-\Theta\right)-\ln \left(\lambda^{+}-1-\Theta\right)\right), \\
& f^{-}(\Theta):=\alpha^{-}\left(\ln \left(\lambda^{-}+\Theta\right)-\ln \left(\lambda^{-}+1+\Theta\right)\right) .
\end{aligned}
$$

Taking derivatives of $f^{+}$and $f^{-}$, we get that $f$ is strictly increasing. Noting that

$$
\lim _{\Theta \downarrow-\lambda^{-}} f(\Theta)=-\infty \text { and } \lim _{\Theta \uparrow \lambda^{+}-1} f(\Theta)=\infty,
$$

there exists a unique $\Theta \in\left(-\lambda^{-}, \lambda^{+}-1\right)$ fulfilling 4.3 .

Hence, a martingale measure, which is an Esscher transform $\mathbb{P}^{\Theta}$, exists if and only if 4.1) is satisfied. In order to find the parameter $\Theta \in\left(-\lambda^{-}, \lambda^{+}-1\right)$, we have to solve equation 4.2. In general, this has to be done numerically. In the particular situation $\alpha^{+}=\alpha^{-}$, which according to [27. Thm. 3.3] is the case if and only if $X$ is Variance Gamma, and $r=q$ the solution for equation (4.2) is given by $\Theta=\frac{1}{2}\left(\lambda^{+}-\lambda^{-}-1\right)$, the midpoint of the interval $\left(-\lambda^{-}, \lambda^{+}-1\right)$. 
4.4. Remark. For general tempered stable distributions condition (4.1) alone is not sufficient for the existence of an Esscher martingale measure. This is due to the fact that the cumulant generating functions of tempered stable distributions, which are not bilateral Gamma, have finite values at the boundaries, which gives rise to an extra condition on the parameters.

\section{EXISTENCE OF MINIMAL ENTROPY MARTINGALE MEASURES IN BILATERAL GAMMA STOCK MODELS}

We have seen in the previous section that in bilateral Gamma stock models an equivalent martingale measure is easy to obtain by solving equation (4.2), provided condition (4.1) is satisfied. Moreover, the driving process $X$ is still a bilateral Gamma process under the new measure, which allows numerical calculations of option prices. However, it is not clear that, in reality, the market chooses this kind of measure.

In the literature, one often performs option pricing by finding an equivalent martingale measure $\mathbb{Q} \sim \mathbb{P}$ which minimizes

$$
\mathbb{E}\left[f\left(\Lambda_{1}(\mathbb{Q}, \mathbb{P})\right)\right]
$$

for a strictly convex function $f:(0, \infty) \rightarrow \mathbb{R}$. Popular choices for the functional $f$ are $f(x)=x^{p}$ for some $p>1$ (see, e.g., [43, 17, 36, 23, 20, 22]) and $f(x)=x \ln x$. In the special case $p=2$ the measure $\mathbb{Q}$ is known as the variance-optimal martingale measure, see, e.g., 41, 7, 32, 33, 44, 4]. It follows from [2, Ex. 2.7] that for bilateral Gamma stock models a $p$-optimal equivalent martingale measure does not exist, because we would need that the tails for upward jumps are extraordinarily light. We can merely obtain the existence of a $p$-optimal signed martingale measure and of a $p$-optimal absolutely continuous martingale measure for our model.

In this section, we shall consider the functional $f(x)=x \ln x$. Then, the quantity

$$
\mathbb{H}(\mathbb{Q} \mid \mathbb{P}):=\mathbb{E}\left[\Lambda_{1}(\mathbb{Q}, \mathbb{P}) \ln \Lambda_{1}(\mathbb{Q}, \mathbb{P})\right]=\mathbb{E}_{\mathbb{Q}}\left[\ln \Lambda_{1}(\mathbb{Q}, \mathbb{P})\right]
$$

is called the relative entropy. In connection with exponential Lévy models, it has been studied, e.g., in [5, 13, 11, 18, see also [25]. The minimal entropy has an information theoretic interpretation: minimizing relative entropy corresponds to choosing a martingale measure by adding the least amount of information to the prior model.

From a mathematical point of view, minimizing the relative entropy is convenient, because there is a connection between the minimal entropy and Esscher transforms of the exponential transform $\tilde{Y}:=\mathcal{L}(\tilde{S})$ of the discounted stock price process $\tilde{S}$, which has rigorously been presented in [1] and further been extended in [18. Note that $\tilde{S}_{t}=S_{0} e^{Y_{t}}$, where $Y$ denotes the Lévy process $Y_{t}:=X_{t}-(r-q) t$.

We shall now recall this connection in our framework, where $X$ is a bilateral Gamma process. By [18, Thm. 2], the exponential transform $\tilde{Y}$ is a Lévy process, which has the characteristic triplet

$$
\begin{aligned}
\tilde{b} & =\int_{\mathbb{R}} h\left(e^{x}-1\right) F(d x)-(r-q), \\
\tilde{c} & =0, \\
\tilde{F}(B) & =\int_{\mathbb{R}} \mathbb{1}_{B}\left(e^{x}-1\right) F(d x), \quad B \in \mathcal{B}(\mathbb{R}) .
\end{aligned}
$$

with respect to the truncation function $h(x)=x \mathbb{1}_{[-1,1]}(x)$, where the Lévy measure $F$ is given by (3.3). Therefore, the cumulant generating function $\tilde{\Psi}$ of $\tilde{Y}$ exists on 
$\mathbb{R}_{-}:=(-\infty, 0]$ and is given by

$$
\begin{aligned}
\tilde{\Psi}(z) & =-(r-q) z+\int_{\mathbb{R}}\left(e^{z x}-1\right) \tilde{F}(d x) \\
& =-(r-q) z+\int_{\mathbb{R}}\left(e^{z\left(e^{x}-1\right)}-1\right) F(d x), \quad z \leq 0 .
\end{aligned}
$$

5.1. Definition. Let $\vartheta \leq 0$ be arbitrary. The Esscher transform $\mathbb{P}_{\vartheta} \stackrel{\text { loc }}{\sim} \mathbb{P}$ is the locally equivalent probability measure with likelihood process

$$
\Lambda_{t}\left(\mathbb{P}_{\vartheta}, \mathbb{P}\right):=\left.\frac{d \mathbb{P}_{\vartheta}}{d \mathbb{P}}\right|_{\mathcal{F}_{t}}=e^{\vartheta \tilde{Y}_{t}-\tilde{\Psi}(\vartheta) t}, \quad t \geq 0 .
$$

The following result, which establishes the connection between the minimal entropy and Esscher transforms, will be crucial for our investigations.

5.2. Proposition. There exists a minimal entropy martingale measure if and only if there exists $\vartheta \leq 0$ such that $\mathbb{E}_{\vartheta}\left[\tilde{Y}_{1}\right]=0$. In this case, one minimal entropy martingale measure is given by $\mathbb{P}_{\vartheta}$.

Proof. The assertion follows from [18, Thm. 8] and Lemma 2.5.

We are now ready to characterize all bilateral Gamma stock models, for which minimal entropy martingale measures exist, and to determine the value of this minimal entropy.

5.3. Theorem. Let $X \sim \Gamma\left(\alpha^{+}, \lambda^{+} ; \alpha^{-}, \lambda^{-}\right)$under $\mathbb{P}$.

(1) If $\lambda^{+} \leq 1$, there exists a unique $\vartheta<0$ such that $\mathbb{P}_{\vartheta}$ is a minimal entropy martingale measure. It is the unique solution of the equation

$$
\begin{aligned}
& \alpha^{+} \int_{0}^{\infty} \frac{1}{x} e^{-\lambda^{+} x}\left(e^{x}-1\right) e^{\vartheta\left(e^{x}-1\right)} d x \\
& +\alpha^{-} \int_{0}^{\infty} \frac{1}{x} e^{-\lambda^{-} x}\left(e^{-x}-1\right) e^{\vartheta\left(e^{-x}-1\right)} d x=r-q, \quad \vartheta \in(-\infty, 0) .
\end{aligned}
$$

(2) If $\lambda^{+}>1$, there exists $\vartheta \leq 0$ such that $\mathbb{P}_{\vartheta}$ is a minimal entropy measure if and only if

$$
\alpha^{+} \ln \left(\frac{\lambda^{+}}{\lambda^{+}-1}\right)+\alpha^{-} \ln \left(\frac{\lambda^{-}}{\lambda^{-}+1}\right) \geq r-q .
$$

If (5.3) is satisfied, $\vartheta$ is unique and it is the unique solution of equation (5.2) for $\vartheta \in \mathbb{R}_{-}$.

(3) If a minimal entropy martingale measure exists, then the value of the minimal entropy is given by

$$
\begin{aligned}
\mathbb{H}\left(\mathbb{P}_{\vartheta} \mid \mathbb{P}\right)= & -\alpha^{+} \int_{0}^{\infty} \frac{1}{x} e^{-\lambda^{+} x}\left(e^{\vartheta\left(e^{x}-1\right)}-1\right) d x \\
& -\alpha^{-} \int_{0}^{\infty} \frac{1}{x} e^{-\lambda^{-} x}\left(e^{\vartheta\left(e^{-x}-1\right)}-1\right) d x+(r-q) \vartheta .
\end{aligned}
$$

Proof. For each $\vartheta \leq 0$ the characteristic triplet of $\tilde{Y}$ with respect to the truncation function $h(x)=x \mathbb{1}_{[-1,1]}(x)$ under the measure $\mathbb{P}_{\vartheta}$ is, according to [18, Thm. 1], given by

$$
\begin{aligned}
\tilde{b}_{\vartheta} & =\tilde{b}+\int_{\mathbb{R}}\left(e^{\vartheta x}-1\right) h(x) \tilde{F}(d x)=\int_{\mathbb{R}} h\left(e^{x}-1\right) e^{\vartheta\left(e^{x}-1\right)} F(d x)-(r-q), \\
\tilde{c}_{\vartheta} & =0, \\
\tilde{F}_{\vartheta}(d x) & =e^{\vartheta x} \tilde{F}(d x) .
\end{aligned}
$$


Hence, we have $\mathbb{E}_{\vartheta}\left|\tilde{Y}_{1}\right|<\infty, \vartheta<0$ and, by 3.3 , the expectations are given by

$$
\begin{aligned}
\mathbb{E}_{\vartheta}\left[\tilde{Y}_{1}\right]= & \int_{\mathbb{R}}\left(e^{x}-1\right) e^{\vartheta\left(e^{x}-1\right)} F(d x)-(r-q) \\
= & \alpha^{+} \int_{0}^{\infty} \frac{1}{x} e^{-\lambda^{+} x}\left(e^{x}-1\right) e^{\vartheta\left(e^{x}-1\right)} d x \\
& +\alpha^{-} \int_{0}^{\infty} \frac{1}{x} e^{-\lambda^{-} x}\left(e^{-x}-1\right) e^{\vartheta\left(e^{-x}-1\right)} d x-(r-q), \quad \vartheta \leq 0 .
\end{aligned}
$$

Moreover, we have $\mathbb{E}_{0}\left|\tilde{Y}_{1}\right|<\infty$ if and only if $\lambda^{+}>1$, and in this case the expectation is, by (3.4), given by

$$
\mathbb{E}_{0}\left[\tilde{Y}_{1}\right]=\Psi(1)-(r-q)=\alpha^{+} \ln \left(\frac{\lambda^{+}}{\lambda^{+}-1}\right)+\alpha^{-} \ln \left(\frac{\lambda^{-}}{\lambda^{-}+1}\right)-(r-q)
$$

According to [37, Lemma 26.4] the cumulant generating function $\tilde{\Psi}$ of $\tilde{Y}$ is of class $C^{\infty}$ on $(-\infty, 0)$, we have $\tilde{\Psi}^{\prime \prime}>0$ on $(-\infty, 0)$ and the first derivative is, by the representation (5.1), given by

$$
\tilde{\Psi}^{\prime}(\vartheta)=-(r-q)+\int_{\mathbb{R}}\left(e^{x}-1\right) e^{\vartheta\left(e^{x}-1\right)} F(d x)=\mathbb{E}_{\vartheta}\left[\tilde{Y}_{1}\right], \quad \vartheta \in(-\infty, 0) .
$$

Note that $\tilde{\Psi}^{\prime}(\vartheta) \downarrow-\infty$ for $\vartheta \downarrow-\infty$.

Let us now consider the situation $\lambda^{+} \leq 1$. Then we have $\tilde{\Psi}^{\prime}(\vartheta) \uparrow \infty$ for $\vartheta \uparrow 0$. Since $\tilde{\Psi}^{\prime}$ is continuous and $\tilde{\Psi}^{\prime \prime}>0$ on $(-\infty, 0)$, Proposition 5.2 yields the first statement.

In the case $\lambda^{+}>1$, an analogous argumentation yields, by taking into account (5.5), the second statement.

If a minimal entropy martingale measure exists, then the value of the minimal entropy is given by

$$
\mathbb{H}\left(\mathbb{P}_{\vartheta} \mid \mathbb{P}\right)=\mathbb{E}_{\vartheta}\left[\ln \Lambda_{1}\left(\mathbb{P}_{\vartheta}, \mathbb{P}\right)\right]=\mathbb{E}_{\vartheta}\left[\vartheta \tilde{Y}_{1}-\tilde{\Psi}(\vartheta)\right]=-\tilde{\Psi}(\vartheta),
$$

which, by (5.1) and (3.3), gives us (5.4).

We emphasize that for bilateral Gamma stock models the value of the minimal entropy in (5.4) can easily be calculated numerically in terms of the parameters $\alpha^{+}, \lambda^{+}, \alpha^{-}, \lambda^{-}$.

5.4. Remark. For tempered stable processes a similar version of Theorem 5.3 holds true. The terms in (5.2) and (5.4) slightly change, namely, the fraction $\frac{1}{x}$ is replaced by $\frac{1}{x^{1+\beta^{+}}}$resp. $\frac{1}{x^{1+\beta^{-}}}$, where $\beta^{+}, \beta^{-} \in(0,1)$ are the additional two parameters. Condition (5.3) differs considerably in the tempered stable case, due to the different form of the cumulant generating function $\Psi$.

\section{Existence of Minimal ENTROPy MARTingale MEASURES PRESERVING THE Class of BILATERAl Gamma PROCESSES}

We have seen in the previous section that for bilateral Gamma stock models we obtain the minimal entropy martingale measure $\mathbb{P}_{\vartheta}$ by solving equation 5.2 numerically, provided $\lambda^{+} \leq 1$ or condition $(5.3)$ is satisfied. Under $\mathbb{P}_{\vartheta}$, the bilateral Gamma process $X$ is still a Lévy process, a result which is due to [11], and we know its characteristic triplet. However, neither its one-dimensional density nor its characteristic function is available in closed form, hence we cannot perform option pricing numerically.

Recall that, on the other hand, the Esscher transform from Section 4 leaves the family of bilateral Gamma processes invariant. 
Our idea in this section is therefore as follows. We minimize the relative entropy within the class of bilateral Gamma processes by performing bilateral Esscher transforms.

6.1. Definition. Let $X \sim \Gamma\left(\alpha^{+}, \lambda^{+} ; \alpha^{-}, \lambda^{-}\right)$under $\mathbb{P}$ and let $\theta^{+} \in\left(-\infty, \lambda^{+}\right)$and $\theta^{-} \in\left(-\infty, \lambda^{-}\right)$be arbitrary. The bilateral Esscher transform $\mathbb{P}^{\left(\theta^{+}, \theta^{-}\right)} \stackrel{\text { loc }}{\sim} \mathbb{P}$ is defined as the locally equivalent probability measure with likelihood process

$$
\Lambda_{t}\left(\mathbb{P}^{\left(\theta^{+}, \theta^{-}\right)}, \mathbb{P}\right):=\left.\frac{d \mathbb{P}^{\left(\theta^{+}, \theta^{-}\right)}}{d \mathbb{P}}\right|_{\mathcal{F}_{t}}=e^{\theta^{+} X_{t}^{+}-\Psi^{+}\left(\theta^{+}\right) t} \cdot e^{\theta^{-} X_{t}^{-}-\Psi^{-}\left(\theta^{-}\right) t}, \quad t \geq 0
$$

where $\Psi^{+}, \Psi^{-}$denote the cumulant generating functions given by (3.5), (3.6).

Note that the Esscher transforms $\mathbb{P}^{\Theta}$ from Section 4 are special cases of the just introduced bilateral Esscher transforms $\mathbb{P}^{\left(\theta^{+}, \theta^{-}\right)}$. Indeed, it holds

$$
\mathbb{P}^{\Theta}=\mathbb{P}^{(\Theta,-\Theta)}, \quad \Theta \in\left(-\lambda^{-}, \lambda^{+}\right) .
$$

6.2. Lemma. Let $X \sim \Gamma\left(\alpha^{+}, \lambda^{+} ; \alpha^{-}, \lambda^{-}\right)$under $\mathbb{P}$ and let $\theta^{+} \in\left(-\infty, \lambda^{+}\right)$and $\theta^{-} \in\left(-\infty, \lambda^{-}\right)$be arbitrary. Then we have $X \sim \Gamma\left(\alpha^{+}, \lambda^{+}-\theta^{+} ; \alpha^{-}, \lambda^{-}-\theta^{-}\right)$ under $\mathbb{P}^{\left(\theta^{+}, \theta^{-}\right)}$.

Proof. This follows from Proposition 2.1.3 and Example 2.1.4 in [26].

According to [27, Prop. 6.1], the parameters $\alpha^{+}$and $\alpha^{-}$cannot be changed to other parameters by an equivalent measure transformation. Consequently, any equivalent measure transformation, under which $X$ is still a bilateral Gamma process, is a bilateral Esscher transform.

6.3. Lemma. Let $X \sim \Gamma\left(\alpha^{+}, \lambda^{+} ; \alpha^{-}, \lambda^{-}\right)$under $\mathbb{P}$ and let $\theta^{+} \in\left(-\infty, \lambda^{+}\right)$and $\theta^{-} \in\left(-\infty, \lambda^{-}\right)$be arbitrary. Then $\mathbb{P}^{\left(\theta^{+}, \theta^{-}\right)}$is a martingale measure if and only if $\theta^{+} \in\left(\lambda^{+}-\left(1-\exp \left(-\frac{r-q}{\alpha^{+}}\right)\right)^{-1}, \lambda^{+}-1\right)$ and

$$
\theta^{-}=\Phi\left(\theta^{+}\right),
$$

where $\Phi:\left(\lambda^{+}-\left(1-\exp \left(-\frac{r-q}{\alpha^{+}}\right)\right)^{-1}, \lambda^{+}-1\right) \rightarrow\left(-\infty, \lambda^{-}\right)$is defined as the strictly increasing function

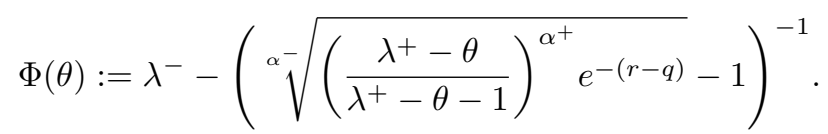

By convention, we set $\lambda^{+}-\left(1-\exp \left(-\frac{r-q}{\alpha^{+}}\right)\right)^{-1}:=-\infty$ if $r=q$.

Proof. This is an immediate consequence of Lemma 6.2 and Lemma 3.1

As pointed out above, all equivalent measure transformations preserving the class of bilateral Gamma processes are bilateral Esscher transforms. Hence, we introduce the set of parameters

$$
\mathcal{M}_{\mathbb{P}}:=\left\{\left(\theta^{+}, \theta^{-}\right) \in\left(-\infty, \lambda^{+}\right) \times\left(-\infty, \lambda^{-}\right) \mid \mathbb{P}^{\left(\theta^{+}, \theta^{-}\right)} \text {is a martingale measure }\right\}
$$

such that the bilateral Esscher transform is a martingale measure. The previous Lemma 6.3 tells us that

$$
\mathcal{M}_{\mathbb{P}}=\left\{(\theta, \Phi(\theta)) \in \mathbb{R}^{2} \mid \theta \in\left(\lambda^{+}-\left(1-\exp \left(-\frac{r-q}{\alpha^{+}}\right)\right)^{-1}, \lambda^{+}-1\right)\right\} .
$$

The following consequence contributes to Theorem 4.3 . It tells us that, provided (4.1) is satisfied, we can, instead of solving (4.2), alternatively solve equation (6.5) below in order to find the Esscher transform $\mathbb{P}^{\Theta}$. 
6.4. Corollary. Let $X \sim \Gamma\left(\alpha^{+}, \lambda^{+} ; \alpha^{-}, \lambda^{-}\right)$under $\mathbb{P}$. If $\left.\sqrt{4.1}\right)$ is satisfied, then the unique $\Theta \in\left(-\lambda^{-}, \lambda^{+}-1\right)$ such that $\mathbb{P}^{\Theta}$ is a martingale measure is the unique solution of the equation

$$
\Phi(\Theta)=-\Theta, \quad \Theta \in\left(\lambda^{+}-\left(1-\exp \left(-\frac{r-q}{\alpha^{+}}\right)\right)^{-1}, \lambda^{+}-1\right) .
$$

Proof. The proof follows from Theorem 4.3 and relations (6.1), (6.4).

We have considered the case $\alpha^{+}=\alpha^{-}$and $r=q$ at the end of Section 4 . In this particular situation $\Phi:\left(-\infty, \lambda^{+}-1\right) \rightarrow\left(-\infty, \lambda^{-}\right)$is given by $\Phi(\theta)=\lambda^{-}-\lambda^{+}+\theta+1$, whence we see again, this time by solving equation (6.5), that the Esscher parameter is given by $\Theta=\frac{1}{2}\left(\lambda^{+}-\lambda^{-}-1\right)$.

We are now ready to treat the existence of minimal entropy martingale measures in bilateral Gamma stock markets, under which $X$ remains a bilateral Gamma process.

6.5. Theorem. Let $X \sim \Gamma\left(\alpha^{+}, \lambda^{+} ; \alpha^{-}, \lambda^{-}\right)$under $\mathbb{P}$ with arbitrary parameters $\alpha^{+}, \lambda^{+}, \alpha^{-}, \lambda^{-}>0$. Then, there exist $\theta^{+} \in\left(-\infty, \lambda^{+}\right)$and $\theta^{-} \in\left(-\infty, \lambda^{-}\right)$such that

$$
\mathbb{H}\left(\mathbb{P}^{\left(\theta^{+}, \theta^{-}\right)} \mid \mathbb{P}\right)=\min _{\left(\vartheta+, \vartheta^{-}\right) \in \mathcal{M}_{\mathbb{P}}} \mathbb{H}\left(\mathbb{P}^{\left(\vartheta^{+}, \vartheta^{-}\right)} \mid \mathbb{P}\right) .
$$

In this case, we have $\theta^{+} \in\left(\lambda^{+}-\left(1-\exp \left(-\frac{r-q}{\alpha^{+}}\right)\right)^{-1}, \lambda^{+}-1\right)$, relation 6.2) is satisfied, and $\theta^{+}$minimizes the function $f:\left(\lambda^{+}-\left(1-\exp \left(-\frac{r-q}{\alpha^{+}}\right)\right)^{-1}, \lambda^{+}-1\right) \rightarrow \mathbb{R}$ defined as

$$
\begin{aligned}
f(\theta):= & \alpha^{+}\left(\frac{\lambda^{+}}{\lambda^{+}-\theta}-1-\ln \left(\frac{\lambda^{+}}{\lambda^{+}-\theta}\right)\right) \\
& +\alpha^{-}\left(\frac{\lambda^{-}}{\lambda^{-}-\Phi(\theta)}-1-\ln \left(\frac{\lambda^{-}}{\lambda^{-}-\Phi(\theta)}\right)\right) .
\end{aligned}
$$

Moreover, we have $X \sim \Gamma\left(\alpha^{+}, \lambda^{+}-\theta^{+} ; \alpha^{-}, \lambda^{-}-\theta^{-}\right)$under $\mathbb{P}^{\left(\theta^{+}, \theta^{-}\right)}$, and the value of the minimal entropy is given by

$$
\mathbb{H}\left(\mathbb{P}^{\left(\theta^{+}, \theta^{-}\right)} \mid \mathbb{P}\right)=f\left(\theta^{+}\right) .
$$

Proof. For $\theta^{+} \in\left(-\infty, \frac{\lambda^{+}}{p}\right)$ and $\theta^{-} \in\left(-\infty, \frac{\lambda^{-}}{p}\right)$ the relative entropy is, by taking into account Lemma 6.2 given by

$$
\begin{aligned}
\mathbb{H}\left(\mathbb{P}^{\left(\theta^{+}, \theta^{-}\right)} \mid \mathbb{P}\right) & =\mathbb{E}_{\mathbb{P}^{\left(\theta^{+}, \theta^{-}\right)}}\left[\ln \left(\frac{d \mathbb{P}^{\left(\theta^{+}, \theta^{-}\right)}}{d \mathbb{P}}\right)\right] \\
& =\mathbb{E}_{\mathbb{P}^{\left(\theta^{+}, \theta^{-}\right)}}\left[\theta^{+} X_{1}^{+}-\Psi^{+}\left(\theta^{+}\right)+\theta^{-} X_{1}^{-}-\Psi^{-}\left(\theta^{-}\right)\right] \\
& =\frac{\theta^{+} \alpha^{+}}{\lambda^{+}-\theta^{+}}-\Psi^{+}\left(\theta^{+}\right)+\frac{\theta^{-} \alpha^{-}}{\lambda^{-}-\theta^{-}}-\Psi^{-}\left(\theta^{-}\right) \\
& =\alpha^{+} g\left(\frac{\lambda^{+}}{\lambda^{+}-\theta^{+}}\right)+\alpha^{-} g\left(\frac{\lambda^{-}}{\lambda^{-}-\theta^{-}}\right),
\end{aligned}
$$

where $g:(0, \infty) \rightarrow \mathbb{R}$ denotes the strictly convex function $g(x)=x-1-\ln x$. Thus, for all $\left(\theta^{+}, \theta^{-}\right) \in \mathcal{M}_{\mathbb{P}}$ the relative entropy $\mathbb{H}\left(\mathbb{P}^{\left(\theta^{+}, \theta^{-}\right)} \mid \mathbb{P}\right)$ is given by 6.8), and we can write the function $f$ as

$$
f(\theta)=\alpha^{+} g\left(\frac{\lambda^{+}}{\lambda^{+}-\theta}\right)+\alpha^{-} g\left(\frac{\lambda^{-}}{\lambda^{-}-\Phi(\theta)}\right) .
$$

Note that

$$
\lim _{\theta \downarrow \lambda^{+}-\left(1-\exp \left(-\frac{r-q}{\alpha+}\right)\right)^{-1}} \frac{\lambda^{+}}{\lambda^{+}-\theta} \in\left[0, \lambda^{+}\right) \quad \text { and } \quad \lim _{\theta \uparrow \lambda^{+}-1} \frac{\lambda^{+}}{\lambda^{+}-\theta}=\lambda^{+}
$$


as well as

$$
\lim _{\theta \downarrow \lambda^{+}-\left(1-\exp \left(-\frac{r-q}{\alpha+}\right)\right)^{-1}} \frac{\lambda^{-}}{\lambda^{-}-\Phi(\theta)}=0 \quad \text { and } \quad \lim _{\theta \uparrow \lambda^{+}-1} \frac{\lambda^{-}}{\lambda^{-}-\Phi(\theta)}=\infty .
$$

We conclude that

$$
\lim _{\theta \downarrow \lambda^{+}-\left(1-\exp \left(-\frac{r-q}{\alpha+}\right)\right)^{-1}} f(\theta)=\infty \quad \text { and } \quad \lim _{\theta \uparrow \lambda^{+}-1} f(\theta)=\infty .
$$

Since $f$ is continuous, it attains a minimum and the assertion follows.

Consequently, unlike the Esscher transform $\mathbb{P}^{\theta}$ from Section 4 and the real minimal entropy martingale measure $\mathbb{P}_{\vartheta}$ from Section 5 , the minimal entropy martingale measure $\mathbb{P}^{(\theta, \Phi(\theta))}$ in the subclass of all measures which leave bilateral Gamma processes invariant, always exists. In this case, it is also possible to determine the minimal entropy numerically by minimizing the function $f$ defined in (6.7). A comparison with the value in (5.4) will be given in a concrete example in Section 8 .

6.6. Remark. If condition (4.1) is satisfied, choosing the Esscher parameter $\Theta \in$ $\left(-\lambda^{-}, \lambda^{+}-1\right)$ from Section 4 and inserting it into the function $f$, we obtain, by Corollary 6.4 and the representation (6.9) of $f$,

$$
\mathbb{H}\left(\mathbb{P}^{\Theta} \mid \mathbb{P}\right)=f(\Theta)=\alpha^{+} g\left(\frac{\lambda^{+}}{\lambda^{+}-\Theta}\right)+\alpha^{-} g\left(\frac{\lambda^{-}}{\lambda^{-}+\Theta}\right) .
$$

Note that one of the arguments in 6.10 for the function $g$ is greater than 1 , whereas the other argument is smaller than 1. Since the strictly convex function $g$ attains its global minimum at $x=1$, and, by Taylor's theorem, behaves like $(x-1)^{2}$ around $x=1$, the relative entropy $\mathbb{H}\left(\mathbb{P}^{\Theta} \mid \mathbb{P}\right)$ in $\sqrt{6.10}$ is, in general, not too far from the minimal relative entropy $\mathbb{H}\left(\mathbb{P}^{(\theta, \Phi(\theta))} \mid \mathbb{P}\right)$ in 6.6$)$. Therefore, we expect that, typically, the Esscher parameter $\Theta$ and the bilateral Esscher parameter $\theta$ are close to each other.

In general, we have the inequalities

$$
\mathbb{H}\left(\mathbb{P}_{\vartheta} \mid \mathbb{P}\right)<\mathbb{H}\left(\mathbb{P}^{(\theta, \Phi(\theta))} \mid \mathbb{P}\right)<\mathbb{H}\left(\mathbb{P}^{\Theta} \mid \mathbb{P}\right),
$$

provided, the respective measures exist. Using our preceding results, we can compute the values of the respective entropies numerically and see, how close they are to each other.

Since $X$ is still a bilateral Gamma process under the bilateral Esscher transform $\mathbb{P}^{(\theta, \Phi(\theta))}$, we can perform option pricing by the method of Fourier transformation.

The characteristic function of a bilateral Gamma distribution is given by (3.1) and all increments of $X$ are bilateral Gamma distributed, see $\sqrt{3.2}$. Therefore, if $\lambda^{+}-\theta>1$, the price of a European call option with strike price $K$ and time of maturity $T$ is given by

$$
\begin{aligned}
\pi\left(S_{0}\right)=-\frac{e^{-r T} K}{2 \pi} & \int_{i \nu-\infty}^{i \nu+\infty}\left(\frac{K}{S_{0}}\right)^{i z}\left(\frac{\lambda^{+}-\theta}{\lambda^{+}-\theta+i z}\right)^{\alpha^{+} T} \\
& \times\left(\frac{\lambda^{-}-\Phi(\theta)}{\lambda^{-}-\Phi(\theta)-i z}\right)^{\alpha^{-} T} \frac{d z}{z(z-i)},
\end{aligned}
$$

where $\nu \in\left(1, \lambda^{+}-\theta\right)$ is arbitrary. This follows from [29, Thm. 3.2].

As mentioned in Section 5, for bilateral Gamma stock models the p-optimal martingale measure does not exist. However, we can, as provided for the minimal entropy martingale measure, determine the $p$-optimal martingale measure within the class of bilateral Gamma processes. 
The $p$-distance of a bilateral Esscher transform is easy to compute. Indeed, since $X^{+}$and $X^{-}$are independent, for $p>1$ and $\theta^{+} \in\left(-\infty, \frac{\lambda^{+}}{p}\right), \theta^{-} \in\left(-\infty, \frac{\lambda^{-}}{p}\right)$ the $p$-distance is given by

$$
\begin{aligned}
& \mathbb{E}\left[\left(\frac{d \mathbb{P}^{\left(\theta^{+}, \theta^{-}\right)}}{d \mathbb{P}}\right)^{p}\right]=e^{-p\left(\Psi^{+}\left(\theta^{+}\right)+\Psi^{-}\left(\theta^{-}\right)\right)} \mathbb{E}\left[e^{p \theta^{+} X_{1}^{+}}\right] \mathbb{E}\left[e^{p \theta^{-} X_{1}^{-}}\right] \\
& =\exp \left(-p\left(\Psi^{+}\left(\theta^{+}\right)+\Psi^{-}\left(\theta^{-}\right)\right)+\Psi^{+}\left(p \theta^{+}\right)+\Psi^{-}\left(p \theta^{-}\right)\right) \\
& =\left(\frac{\lambda^{+}}{\lambda^{+}-p \theta^{+}}\right)^{\alpha^{+}}\left(\frac{\lambda^{-}}{\lambda^{-}-p \theta^{-}}\right)^{\alpha^{-}}\left(\frac{\lambda^{+}-\theta^{+}}{\lambda^{+}}\right)^{p \alpha^{+}}\left(\frac{\lambda^{-}-\theta^{-}}{\lambda^{-}}\right)^{p \alpha^{-}} .
\end{aligned}
$$

A similar argumentation as in Theorem 6.5 shows that, for each choice of the parameters $\alpha^{+}, \lambda^{+}, \alpha^{-}, \lambda^{-}>0$ there exists a pair $\left(\theta^{+}, \theta^{-}\right)$minimizing the $p$-distance $(6.12)$, and in this case we also have $(6.2)$, where $\theta^{+}$minimizes the function

$$
f_{p}(\theta)=\left(\frac{\lambda^{+}}{\lambda^{+}-p \theta}\right)^{\alpha^{+}}\left(\frac{\lambda^{-}}{\lambda^{-}-p \Phi(\theta)}\right)^{\alpha^{-}}\left(\frac{\lambda^{+}-\theta}{\lambda^{+}}\right)^{p \alpha^{+}}\left(\frac{\lambda^{-}-\Phi(\theta)}{\lambda^{-}}\right)^{p \alpha^{-}}
$$

As we shall see in the numerical illustration of the upcoming section, we have $\theta_{p} \rightarrow \theta$ for $p \downarrow 1$, where for each $p>1$ the parameter $\theta_{p}$ minimizes 6.13 and $\theta$ minimizes (6.7). This is not surprising, since it is known that, under suitable technical conditions, the $p$-optimal martingale measure converges to the minimal entropy martingale measure for $p \downarrow 1$, see, e.g., [15, 16, 35, 20, 2, 24. Here, we shall only give an intuitive argument. For $\alpha, \lambda>0$ consider the functions

$$
\begin{aligned}
h(\theta) & =\alpha\left[\frac{\lambda}{\lambda-\theta}-1-\ln \left(\frac{\lambda}{\lambda-\theta}\right)\right], \\
h_{p}(\theta) & =\left(\frac{\lambda}{\lambda-p \theta}\right)^{\alpha}\left(\frac{\lambda-\theta}{\lambda}\right)^{p \alpha} \text { for } p>1 .
\end{aligned}
$$

Note that $h$ and $h_{p}$ have a global minimum at $\theta=0$. Moreover, if $p>1$ is close to 1 , then the functions $(p-1) h(\theta)$ and $\ln h_{p}(\theta)$ are very close to each other. Indeed, Taylor's theorem shows that, for an appropriate $\xi \in \mathbb{R}$, which is between 1 and $\frac{\lambda-p \theta}{\lambda-\theta}$, we have

$$
\begin{aligned}
& \left|(p-1) h(\theta)-\ln h_{p}(\theta)\right| \\
& =\alpha\left|(p-1)\left[\frac{\lambda}{\lambda-\theta}-1-\ln \left(\frac{\lambda}{\lambda-\theta}\right)\right]-\ln \left(\frac{\lambda}{\lambda-p \theta}\right)+p \ln \left(\frac{\lambda}{\lambda-\theta}\right)\right| \\
& =\alpha\left|\frac{(p-1) \theta}{\lambda-\theta}+\ln \left(\frac{\lambda-p \theta}{\lambda-\theta}\right)\right| \\
& =\alpha\left|\frac{(p-1) \theta}{\lambda-\theta}+\left[\frac{\lambda-p \theta}{\lambda-\theta}-1-\frac{1}{2 \xi^{2}}\left(\frac{\lambda-p \theta}{\lambda-\theta}-1\right)^{2}\right]\right|=\frac{\alpha}{2 \xi^{2}}\left(\frac{\lambda-p \theta}{\lambda-\theta}-1\right)^{2} .
\end{aligned}
$$

For $p>1$ close to 1 , the expression $\frac{\lambda-p \theta}{\lambda-\theta}$ is close to 1 for all relevant $\theta$. Intuitively, this explains the convergence $\theta_{p} \rightarrow \theta$ for $p \downarrow 1$, which implies the convergence of the $p$-optimal martingale measure to the minimal entropy martingale measure.

6.7. Remark. For tempered stable processes, which are not bilateral Gamma, the situation in this section becomes more involved. It may happen that $\mathcal{M}_{\mathbb{P}}=\emptyset$, i.e. no bilateral Esscher martingale measure exists, which is again due to the behaviour of the cumulant generating function at its boundary. Consequently, the statement of Theorem 6.5 does not hold true in this case. We require an extra condition on the parameters in order to ensure the existence of a minimal entropy martingale measure or of a p-optimal martingale measure. We emphasize that for tempered stable processes the function $\Phi$ defined in (6.3) is no longer available in closed form, which complicates the minimizing procedures of this section for concrete examples. 


\section{Existence of the minimal martingale measure in bilateral Gamma STOCK MODELS}

In this section, we deal with the existence of the minimal martingale measure in bilateral Gamma stock models. The minimal martingale measure was introduced in 12 with the motivation of constructing optimal hedging strategies. Throughout this section, we fix a finite time horizon $T>0$ and assume that $\lambda^{+}>2$. This allows us to define the finite value

$$
c=c\left(\alpha^{+}, \alpha^{-}, \lambda^{+}, \lambda^{-}, r, q\right)=\frac{\Psi(1)-(r-q)}{\Psi(2)-2 \Psi(1)},
$$

where $\Psi$ denotes the cumulant generating function defined in (3.4). Note that

$$
\Psi(2)-2 \Psi(1)=\int_{\mathbb{R}}\left(e^{2 x}-1\right) F(d x)-2 \int_{\mathbb{R}}\left(e^{x}-1\right) F(d x)=\int_{\mathbb{R}}\left(e^{x}-1\right)^{2} F(d x)>0,
$$

whence (7.1) is well-defined. For technical reasons, we shall also assume that the filtration $\left(\mathcal{F}_{t}\right)_{t \geq 0}$ is generated by the bilateral Gamma process $X$.

In order to define the minimal martingale measure, we require the canonical decomposition of the discounted stock prices $\tilde{S}$ defined in 1.2 .

7.1. Lemma. The process $\tilde{S}$ is a special semimartingale with canonical decomposition

$$
\tilde{S}_{t}=S_{0}+M_{t}+A_{t}, \quad t \in[0, T]
$$

where $M$ and $A$ denote the processes

$$
\begin{aligned}
M_{t} & =\int_{0}^{t} \int_{\mathbb{R}} \tilde{S}_{s-}\left(e^{x}-1\right)\left(\mu^{X}(d s, d x)-F(d x) d s\right), \quad t \in[0, T] \\
A_{t} & =(\Psi(1)-(r-q)) \int_{0}^{t} \tilde{S}_{s} d s, \quad t \in[0, T] .
\end{aligned}
$$

The process $M$ is a square-integrable martingale and the finite variation part $A$ has the representation

$$
A_{t}=\int_{0}^{t} \alpha_{s} d\langle M\rangle_{s}, \quad t \in[0, T]
$$

where $\alpha$ denotes the predictable process

$$
\alpha_{t}=\frac{c}{\tilde{S}_{t-}}, \quad t \in[0, T]
$$

7.2. Remark. In (7.4), $\mu^{X}$ denotes the random measure associated to the jumps of $X$, and in (7.6) the process $\langle M\rangle$ denotes the predictable quadratic variation of $M$, i.e., the unique predictable process with paths of finite variation such that $M^{2}-\langle M\rangle$ is a local martingale, see, e.g., [19, Sec. I.4a].

Proof. Note that we can express the discounted stock prices 1.2 as

$$
\tilde{S}_{t}=e^{Y_{t}}, \quad t \in[0, T]
$$

where $Y$ denotes the process

$$
Y_{t}=X_{t}-(r-q) t, \quad t \in[0, T]
$$


Using Itô's formula [19, Thm. I.4.57] we obtain

$$
\begin{aligned}
\tilde{S}_{t}= & S_{0}+\int_{0}^{t} e^{Y_{s-}} d Y_{s}+\sum_{s \leq t}\left(e^{Y_{s}}-e^{Y_{s}-}-e^{Y_{s-}} \Delta Y_{s}\right) \\
= & S_{0}+\int_{0}^{t} \int_{\mathbb{R}} x \tilde{S}_{s-} \mu^{X}(d s, d x)-(r-q) \int_{0}^{t} \tilde{S}_{s} d s \\
& +\int_{0}^{t} \int_{\mathbb{R}} \tilde{S}_{s-}\left(e^{x}-1-x\right) \mu^{X}(d s, d x) \\
= & S_{0}+\int_{0}^{t} \int_{\mathbb{R}} \tilde{S}_{s-}\left(e^{x}-1\right)\left(\mu^{X}(d s, d x)-F(d x) d s\right) \\
& +\left(\int_{\mathbb{R}}\left(e^{x}-1\right) F(d x)-(r-q)\right) \int_{0}^{t} \tilde{S}_{s} d s
\end{aligned}
$$

and hence, $\tilde{S}$ is a special semimartingale with canonical decomposition 7.3 . Recalling that $\lambda^{+}>2$, the process $M$ is a square-integrable martingale, because by (7.2), 3.2 and (3.4) we have

$$
\begin{aligned}
& \mathbb{E}\left[\int_{0}^{T} \int_{\mathbb{R}}\left|\tilde{S}_{s}\left(e^{x}-1\right)\right|^{2} F(d x) d s\right]=\left(\int_{\mathbb{R}}\left(e^{x}-1\right)^{2} F(d x)\right)\left(\int_{0}^{T} \mathbb{E}\left[\tilde{S}_{s}^{2}\right] d s\right) \\
& =S_{0}(\Psi(2)-2 \Psi(1)) \int_{0}^{T} e^{-(r-q) s} \mathbb{E}\left[e^{X_{s}}\right] d s \\
& =S_{0}(\Psi(2)-2 \Psi(1)) \int_{0}^{T} e^{-(r-q) s}\left(\frac{\lambda^{+}}{\lambda^{+}-1}\right)^{\alpha^{+} s}\left(\frac{\lambda^{-}}{\lambda^{-}+1}\right)^{\alpha^{-} s} d s<\infty .
\end{aligned}
$$

Using [19, Thm. II.1.33.a], the predictable quadratic variation is given by

$$
\langle M\rangle_{t}=\left(\int_{\mathbb{R}}\left(e^{x}-1\right)^{2} F(d x)\right) \int_{0}^{t} \tilde{S}_{s}^{2} d s=(\Psi(2)-2 \Psi(1)) \int_{0}^{t} \tilde{S}_{s}^{2} d s, \quad t \in[0, T] .
$$

Therefore, we deduce 7.6 .

Lemma 7.1 shows that $\tilde{S}$ satisfies the so-called structure condition (SC) from [40]. Therefore, according to [40, Prop. 2], the stochastic exponential

$$
\hat{Z}_{t}=\mathcal{E}\left(-\int_{0}^{\bullet} \alpha_{s} d M_{s}\right)_{t}, \quad t \in[0, T]
$$

is a martingale density for $\tilde{S}$, that is $\hat{Z}$ and $\hat{Z} \tilde{S}$ are local $\mathbb{P}$-martingales and $\mathbb{P}\left(\hat{Z}_{0}=\right.$ $1)=1$. The measure transformation

$$
\frac{d \hat{\mathbb{P}}}{d \mathbb{P}}:=\hat{Z}_{T}
$$

defines a (possibly signed) measure $\hat{\mathbb{P}}$, the so-called minimal martingale measure for $\tilde{S}$.

We are now interested in the question when $\hat{Z}$ is a strict martingale density, that is, the martingale density $\hat{Z}$ is strictly positive. In this case, the martingale density $\hat{Z}$ is a strictly positive supermartingale, and hence the transformation 7.9 defines (after normalizing, if necessary) a probability measure. If $\hat{Z}$ is even a strictly positive $\mathbb{P}$-martingale, then $\hat{\mathbb{P}}$ is a martingale measure for $\tilde{S}$.

7.3. Theorem. The following statements are equivalent.

(1) $\hat{Z}$ is a strict martingale density for $\tilde{S}$.

(2) $\hat{Z}$ is a strictly positive $\mathbb{P}$-martingale. 
(3) We have

$$
-1 \leq c \leq 0
$$

(4) We have

$$
\begin{aligned}
&\left(\frac{\lambda^{+}}{\lambda^{+}-1}\right)^{\alpha^{+}}\left(\frac{\lambda^{-}}{\lambda^{-}+1}\right)^{\alpha^{-}} \leq e^{r-q} \\
& \text { and } \quad\left(\frac{\lambda^{+}-2}{\lambda^{+}-1}\right)^{\alpha^{+}}\left(\frac{\lambda^{-}+2}{\lambda^{-}+1}\right)^{\alpha^{-}} \leq e^{-(r-q)} .
\end{aligned}
$$

If the previous conditions are satisfied, then under the minimal martingale measure $\hat{\mathbb{P}}$ we have

$$
X \sim \Gamma\left((c+1) \alpha^{+}, \lambda^{+} ;(c+1) \alpha^{-}, \lambda^{-}\right) * \Gamma\left(-c \alpha^{+}, \lambda^{+}-1 ;-c \alpha^{-}, \lambda^{-}+1\right) .
$$

7.4. Remark. Relation (7.13) means that under $\hat{\mathbb{P}}$ the driving process $X$ is the sum of two independent bilateral Gamma processes, that is, the characteristic function of $X_{1}$ under $\hat{\mathbb{P}}$ is given by

$$
\begin{aligned}
\hat{\phi}(z)= & \left(\frac{\lambda^{+}}{\lambda^{+}-i z}\right)^{(c+1) \alpha^{+}}\left(\frac{\lambda^{-}}{\lambda^{-}+i z}\right)^{(c+1) \alpha^{-}} \\
& \times\left(\frac{\lambda^{+}-1}{\lambda^{+}-1-i z}\right)^{-c \alpha^{+}}\left(\frac{\lambda^{-}+1}{\lambda^{-}+1+i z}\right)^{-c \alpha^{-}}, \quad z \in \mathbb{R} .
\end{aligned}
$$

There are the following two boundary values.

- In the case $c=0$ we have

$$
X \sim \Gamma\left(\alpha^{+}, \lambda^{+}, \alpha^{-}, \lambda^{-}\right) \quad \text { under } \hat{\mathbb{P}}
$$

i.e., the minimal martingale measure $\hat{\mathbb{P}}$ coincides with the physical measure $\mathbb{P}$. Indeed, the definition (7.1) of $c$ and Lemma 3.1 show that $\mathbb{P}$ already is a martingale measure for $\bar{S}$.

- In the case $c=-1$ we have

$$
X \sim \Gamma\left(\alpha^{+}, \lambda^{+}-1, \alpha^{-}, \lambda^{-}+1\right) \text { under } \hat{\mathbb{P}},
$$

i.e., the minimal martingale measure $\hat{\mathbb{P}}$ coincides with the Esscher transform $\mathbb{P}^{1}$, see Theorem 4.3. Indeed, the definition (7.1) of c shows that equation (4.2) is satisfied with $\Theta=1$.

Proof. According to [40, Prop. 2] the process $\hat{Z}$ is a strict martingale density for $\tilde{S}$ if and only if we have almost surely

$$
\alpha_{t} \Delta M_{t}<1, \quad t \in[0, T] .
$$

Taking into account Lemma 7.1, this is satisfied if and only if we have almost surely

$$
c\left(e^{\Delta X_{t}}-1\right)<1, \quad t \in[0, T]
$$

and this is the case if and only if we have 7.10 . Noting 7.2 , we observe that (7.10 is equivalent to the two conditions

$$
\Psi(1) \leq r-q \quad \text { and } \quad \Psi(1)-\Psi(2) \leq-(r-q),
$$

and, in view of the cumulant generating function given by $(3.4)$, these two conditions are fulfilled if and only if we have (7.11) and (7.12).

Now suppose that 7.10 is satisfied. We define the continuous functions

$$
\begin{aligned}
& Y: \mathbb{R} \rightarrow(0, \infty), \quad Y(x):=c+1-c e^{x} \\
& f:(0, \infty) \rightarrow \mathbb{R}_{+}, \quad f(y):=y \ln y-(y-1) .
\end{aligned}
$$


Then, by Lemma 7.1. we can write the density process 7.8 as

$$
\begin{aligned}
\hat{Z}_{t} & =\mathcal{E}\left(-\int_{0}^{\bullet} \alpha_{s} d M_{s}\right)_{t}=\mathcal{E}\left(\int_{0}^{\bullet} \int_{\mathbb{R}}\left(c-c e^{x}\right)\left(\mu^{X}(d s, d x)-F(d x) d s\right)\right)_{t} \\
& =\mathcal{E}\left(\int_{0}^{\bullet} \int_{\mathbb{R}}(Y(x)-1)\left(\mu^{X}(d s, d x)-F(d x) d s\right)\right)_{t}, \quad t \in[0, T] .
\end{aligned}
$$

Note that $Y$ is nondecreasing with $Y(0)=1$. Therefore, we have

$$
\int_{\mathbb{R}} f(Y(x)) F(d x) \leq \int_{-\infty}^{0}(1-Y(x)) F(d x)+\int_{0}^{\infty} Y(x) \ln Y(x) F(d x) .
$$

The first term is estimated as

$$
\int_{-\infty}^{0}(1-Y(x)) F(d x)=-c \int_{-\infty}^{0}\left(1-e^{x}\right) F(d x)<\infty .
$$

Taking into account the estimates

$$
\begin{aligned}
Y(x) & \leq 1+e^{x}, \quad x \in \mathbb{R} \\
\ln (1+x) & \leq x, \quad x \geq 0
\end{aligned}
$$

we obtain, since $\lambda^{+}>2$ by assumption,

$$
\begin{aligned}
& \int_{0}^{\infty} Y(x) \ln Y(x) F(d x) \leq \int_{0}^{\infty}\left(e^{x}+1\right) \ln \left(1+c\left(1-e^{x}\right)\right) F(d x) \\
& \leq-c \int_{0}^{\infty}\left(e^{x}+1\right)\left(e^{x}-1\right) F(d x)=-c \int_{0}^{\infty}\left(e^{2 x}-1\right) F(d x)<\infty .
\end{aligned}
$$

Consequently, we have

$$
\int_{\mathbb{R}} f(Y(x)) F(d x)<\infty .
$$

By [11, Cor. 6] the process $\hat{Z}$ is a strictly positive $\mathbb{P}$-martingale. Moreover, [11, Prop. 7] yields that $\beta=0$ and $Y$ are the Girsanov parameters of $\hat{\mathbb{P}}$. Now [11, Prop. 2] gives us that $X$ is a Lévy process under $\hat{\mathbb{P}}$ whose characteristic triplet with respect to the truncation function $h \equiv 0$ is given by $(0,0, \hat{F})$, where, by taking into account (3.3), the Lévy measure is given by

$$
\begin{aligned}
\hat{F}(d x)= & Y(x) F(d x)=\left(c+1-c e^{x}\right) F(d x) \\
= & {\left[\left(\frac{(c+1) \alpha^{+}}{x} e^{-\lambda^{+} x}+\frac{-c \alpha^{+}}{x} e^{-\left(\lambda^{+}-1\right) x}\right) \mathbb{1}_{(0, \infty)}(x)\right.} \\
& \left.+\left(\frac{(c+1) \alpha^{-}}{|x|} e^{-\lambda^{-}|x|}+\frac{-c \alpha^{-}}{|x|} e^{-\left(\lambda^{-}+1\right)|x|}\right) \mathbb{1}_{(-\infty, 0)}(x)\right] d x .
\end{aligned}
$$

Thus, the distribution of $X$ under $\hat{\mathbb{P}}$ is given by 7.13 .

We proceed with an application to quadratic hedging. Here we assume that $\lambda^{+}>3$, which ensures that $\hat{\Psi}(1)$ and $\hat{\Psi}(2)$ appearing in 7.16 below are welldefined. Let $\Phi: \mathbb{R} \rightarrow \mathbb{R}$ be a payoff function. The prices at time $t \in[0, T]$ are given by $\pi\left(t, S_{t}\right)$, where

$$
\pi(t, S)=\mathbb{E}_{\hat{\mathbb{P}}}\left[\Phi\left(S_{T}\right) \mid S_{t}=S\right]
$$


For example, the price at time $t$ of a European call option with strike price $K$ and maturity $T$ is given by

$$
\begin{aligned}
& \pi(t, S)=-\frac{e^{-r(T-t)} K}{2 \pi} \int_{i \nu-\infty}^{i \nu+\infty}\left(\frac{K}{S}\right)^{i z}\left(\frac{\lambda^{+}}{\lambda^{+}+i z}\right)^{(c+1) \alpha^{+}(T-t)} \\
& \quad \times\left(\frac{\lambda^{-}}{\lambda^{-}-i z}\right)^{(c+1) \alpha^{-}(T-t)}\left(\frac{\lambda^{+}-1}{\lambda^{+}-1+i z}\right)^{-c \alpha^{+}(T-t)} \\
& \times\left(\frac{\lambda^{-}+1}{\lambda^{-}+1-i z}\right)^{-c \alpha^{-}(T-t)} \frac{d z}{z(z-i)}
\end{aligned}
$$

where $\nu \in\left(1, \lambda^{+}-1\right)$ is arbitrary. This follows from the form of the characteristic function (7.14) and [29, Thm. 3.2].

Following [6, Sec. 10.4], we choose our trading strategy $\xi$ as

$$
\xi_{t}=\Delta\left(t, S_{t}\right), \quad t \in[0, T]
$$

with $\Delta$ given by

$$
\begin{aligned}
\Delta(t, S) & =\frac{\int_{\mathbb{R}}\left(e^{x}-1\right)\left(\pi\left(t, S e^{x}\right)-\pi(t, S)\right) \hat{F}(d x)}{S \int_{\mathbb{R}}\left(e^{x}-1\right)^{2} \hat{F}(d x)} \\
& =\frac{\int_{\mathbb{R}}\left(e^{x}-1\right)\left(\pi\left(t, S e^{x}\right)-\pi(t, S)\right) \hat{F}(d x)}{S(\hat{\Psi}(2)-2 \hat{\Psi}(1))},
\end{aligned}
$$

where $\hat{F}$ denotes the Lévy measure derived in 7.15 and $\hat{\Psi}$ denotes the cumulant generating function of $X_{1}$ under $\hat{\mathbb{P}}$ given by

$$
\begin{aligned}
\hat{\Psi}(z)= & (c+1) \alpha^{+} \ln \left(\frac{\lambda^{+}}{\lambda^{+}-z}\right)+(c+1) \alpha^{-} \ln \left(\frac{\lambda^{-}}{\lambda^{-}+z}\right) \\
& -c \alpha^{+} \ln \left(\frac{\lambda^{+}-1}{\lambda^{+}-1-z}\right)-c \alpha^{-} \ln \left(\frac{\lambda^{-}+1}{\lambda^{-}+1+z}\right), \quad z \in\left(-\lambda^{-}, \lambda^{+}-1\right) .
\end{aligned}
$$

Note that the strategy $\xi$ is, in general, not self-financing. It minimizes the hedging error

$$
\mathbb{E}_{\hat{\mathbb{P}}}\left[\left|\Phi\left(S_{T}\right)-\left(\mathbb{E}_{\hat{\mathbb{P}}}\left[\Phi\left(S_{T}\right)\right]+\int_{0}^{T} \xi_{t} d S_{t}\right)\right|^{2}\right]
$$

with respect to the $L^{2}(\hat{\mathbb{P}})$-distance. Therefore, we have

$$
\Phi\left(S_{T}\right)=\mathbb{E}_{\hat{\mathbb{P}}}\left[\Phi\left(S_{T}\right)\right]+\int_{0}^{T} \xi_{t} d S_{t}+N_{T},
$$

where $N$ is a local martingale such that $\int_{0}^{T} \xi_{t} d S_{t}$ and $N_{T}$ are orthogonal in $L^{2}(\hat{\mathbb{P}})$. The decomposition (7.17) is the so-called Fölmer-Schweizer decomposition. The orthogonal component $N$ is the intrinsic risk which cannot be hedged. Since the measure change from $\hat{\mathbb{P}}$ to $\mathbb{P}$ preserves orthogonality, $\int_{0}^{T} \xi_{t} d S_{t}$ and $N_{T}$ are also orthogonal in $L^{2}(\mathbb{P})$. Therefore, the strategy $\xi$ also minimizes the quadratic hedging error

$$
\mathbb{E}_{\mathbb{P}}\left[\left|\Phi\left(S_{T}\right)-\left(\mathbb{E}_{\hat{\mathbb{P}}}\left[\Phi\left(S_{T}\right)\right]+\int_{0}^{T} \xi_{t} d S_{t}\right)\right|^{2}\right]
$$

with respect to the physical probability measure $\mathbb{P}$.

7.5. Remark. Analogous results of this section are also valid for general tempered stable processes. The concrete conditions (7.11), (7.12) on the parameters differ considerably in the tempered stable case, due to the different form of the cumulant generating function $\Psi$. 


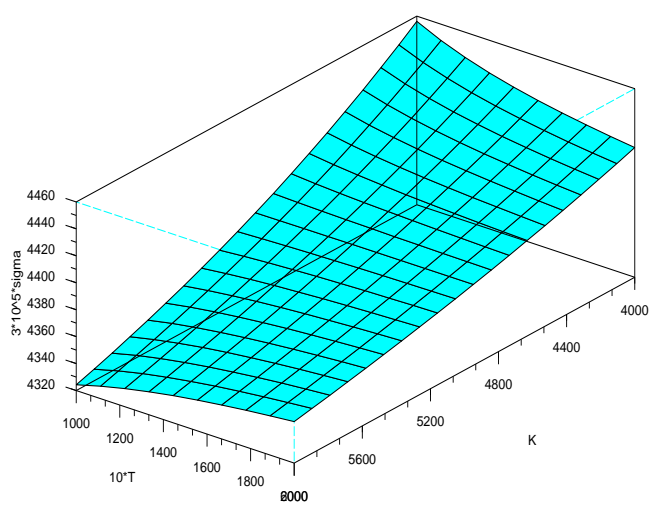

FIGURE 1. Implied volatility surface.

\section{A NUMERICAL ILLUSTRATion}

We conclude this article with a numerical illustration of the preceding results. For a bilateral Gamma stock model of the type (1.1) we have estimated the parameters of the bilateral Gamma process $X$ as

$$
\left(\alpha^{+}, \lambda^{+} ; \alpha^{-}, \lambda^{-}\right)=(1.55,133.96 ; 0.94,88.92)
$$

from observations of the German stock index DAX, see [27, Sec. 9]. For our upcoming calculations, we take the initial stock price $S_{0}=5000$ and, for simplicity, we put $r=q=0$.

We start with the computation of the Esscher transform from Section 4. Note that condition (4.1) is fulfilled. Solving equation (4.2), we obtain the Esscher transform $\mathbb{P}^{\Theta}$ with $\Theta=-5.28$. Under the measure $\mathbb{P}^{\Theta}$, the driving process $X$ is bilateral Gamma with parameters

$$
\left(\alpha^{+}, \lambda^{+}-\Theta ; \alpha^{-}, \lambda^{-}+\Theta\right)=(1.55,139.24 ; 0.94,83.64) .
$$

The value of the relative entropy, provided by $(6.10)$, is given by

$$
\mathbb{H}\left(\mathbb{P}^{\Theta} \mid \mathbb{P}\right)=0.00294113 .
$$

We proceed with the minimal entropy martingale measure from Section 5 . We have

$$
\alpha^{+} \ln \left(\frac{\lambda^{+}}{\lambda^{+}-1}\right)+\alpha^{-} \ln \left(\frac{\lambda^{-}}{\lambda^{-}+1}\right)=0.0000221,
$$

whence condition $(5.3)$ is satisfied. Solving $(5.2)$, we obtain the minimal entropy martingale measure $\mathbb{P}_{\vartheta}$ with $\vartheta=-5.30$. Using (5.4), the value of the minimal entropy is given by

$$
\mathbb{H}\left(\mathbb{P}_{\vartheta} \mid \mathbb{P}\right)=0.00294091
$$

Finally, we turn to the computation of the bilateral Esscher transform from Section 6. Minimizing the function $f$ given by (6.7), we obtain the bilateral Esscher transform $\mathbb{P}^{(\theta, \Phi(\theta))}$ with $\theta=-5.34$. We observe that the Esscher parameter $\Theta$ and the bilateral Esscher parameter $\theta$ are quite close to each other, see Remark 6.6. Under the measure $\mathbb{P}^{(\theta, \Phi(\theta))}$, the driving process $X$ is bilateral Gamma with parameters

$$
\left(\alpha^{+}, \lambda^{+}-\theta ; \alpha^{-}, \lambda^{-}-\Phi(\theta)\right)=(1.55,139.30 ; 0.94,83.68) .
$$


Computing the relative entropy according to 6.8 yields

$$
\mathbb{H}\left(\mathbb{P}^{(\theta, \Phi(\theta))} \mid \mathbb{P}\right)=0.00294107 \text {. }
$$

The relative entropies computed in 8.1), (8.3), 8.4) show that both, the Esscher transform $\mathbb{P}^{\Theta}$ and the bilateral Esscher transform $\mathbb{P}^{(\theta, \Phi(\theta))}$ are quite close to the minimal entropy martingale measure $\mathbb{P}_{\vartheta}$.

For $p>1$ we can compute the $p$-optimal martingale measure within the class of bilateral Gamma processes by minimizing the function $f_{p}$ given by 6.13. In particular, we obtain the variance-optimal martingale measure as the bilateral Esscher transform $\mathbb{P}^{\left(\theta_{2}, \Phi\left(\theta_{2}\right)\right)}$ with $\theta_{2}=-5.68$. Furthermore, we observe that $\theta_{p} \rightarrow \theta$ for $p \downarrow 1$, a behaviour, which we have discussed at the end of Section 6 .

The minimal martingale measure from Section 7 does not exist for the present parameters, because the constant $c$ defined in (7.1) is positive. However, introducing a small interest rate, e.g. we could take $r=0.0012$, then condition 7.10 is fulfilled, and hence the minimal martingale measure $\hat{\mathbb{P}}$ exists.

Going back to the minimal entropy martingale measure $\mathbb{P}^{(\theta, \Phi(\theta))}$, we can numerically compute the prices of European call options by using formula 6.11. Figure 1 shows the implied volatility surface. We observe the following properties:

- The dependence of the implied volatility with respect to the strike price $K$ is decreasing, we have a so-called "skew".

- The skew flattens out for large times of maturity $T$.

Hence, the implied volatility surface in Figure 1 has the typical features, which one observes in practice.

For any positive integer $n \in \mathbb{N}$ we can express $Y \sim \Gamma\left(n \alpha^{+}, \lambda^{+} ; n \alpha^{-}, \lambda^{-}\right)$as $Y=X_{1}+\ldots+X_{n}$, where the $X_{i}$ are i.i.d. with $X_{i} \sim \Gamma\left(\alpha^{+}, \lambda^{+}, \alpha^{-}, \lambda^{-}\right)$. Therefore, if the parameters are fixed, a random variable $X \sim \Gamma\left(\alpha^{+} t, \lambda^{+}, \alpha^{-} t, \lambda^{-}\right)$has the tendency to be approximately normally distributed for increasing $t$, and therefore the implied volatility surface becomes flatter with increasing $t$. This observation also explains why the skew in Figure 1 flattens out for large times of maturity $T$.

Acknowledgement. The second author gratefully acknowledges the support from WWTF (Vienna Science and Technology Fund).

We are grateful to Damir Filipović, Friedrich Hubalek, Katja Krol, Michael Kupper and Antonis Papapantoleon for their helpful remarks and discussions. We also thank two anonymous referees for their helpful comments and suggestions.

\section{REFERENCES}

[1] Barndorff-Nielsen, O. E. (1995) Normal inverse Gaussian distributions and the modeling of stock returns. Research Report no. 300, Department of Theoretical Statistics, Aarhus University.

[2] Bender, C. and Niethammer, C. (2008) On $q$-optimal martingale measures in exponential Lévy models. Finance and Stochastics 12, 381-410.

[3] Carr, P., Geman, H., Madan, D. and Yor, M. (2002) The fine structure of asset returns: an empirical investigation. Journal of Business 75(2), 305-332.

[4] Cerny, A. and Kallsen, J. (2008) A counterexample concerning the variance-optimal martingale measure. Mathematical Finance 18(2), 305-316.

[5] Chan, T. (1999) Pricing contingent claims on stocks driven by Lévy processes. Annals of Applied Probability 9(2), 504-528

[6] Cont, R. and Tankov, P. (2004) Financial modelling with jump processes. Chapman and Hall / CRC Press, London.

[7] Delbaen, F. and Schachermayer, W. (1996) The variance-optimal martingale measure for continuous processes. Bernoulli 2(1), 81-105.

[8] Eberlein, E. and Keller, U. (1995) Hyperbolic distributions in finance. Bernoulli 1, 281-299.

[9] Eberlein, E., Keller, U. and Prause, K. (1998) New insights into smile, mispricing and value at risk: the hyperbolic model. Journal of Business 71, 371-406 
[10] Eberlein, E. and Prause, K. (2001) The generalized hyperbolic model: financial derivatives and risk measures. In: Geman, H., Madan, S., Pliska, S. and Vorst, T. (Eds.), pp. 105-153. Mathematical Finance - Bachelier Congress 2000 (Springer, Berlin).

[11] Esche, F. and Schweizer, M. (2005) Minimal entropy preserves the Lévy property: How and why. Stochastic Processes and Their Applications 115(2), 299-327.

[12] Föllmer, H. and Schweizer, M. (1991) Hedging of contingent claims under incomplete information. In: M. H. A. Davis and R. J. Elliott (eds.), "Applied Stochastic Analysis", Stochastics Monographs, vol. 5, Gordon and Breach, London/New York, 389-414

[13] Fujiwara, T. and Miyahara, Y. (2003) The minimal entropy martingale measures for geometric Lévy processes. Finance and Stochastics 7(4), 509-531.

[14] Gerber, H. U. and Shiu, E. S. W. (1994) Option pricing by Esscher transforms. Trans. Soc. Actuar. XLVI, 98-140.

[15] Grandits, P. (1999) The $p$-optimal martingale measure and its asymptotic relation with the minimal-entropy martingale measure. Bernoulli 5(2), 225-247.

[16] Grandits, P. and Rheinländer, T. (2002) On the minimal entropy martingale measure. Annals of Probability 30(3), 1003-1038.

[17] Hobson, D. (2004) Stochastic volatility models, correlation, and the $q$-optimal measure. Mathematical Finance 14(4), 537-556.

[18] Hubalek, F. and Sgarra, C. (2006) Esscher transforms and the minimal entropy martingale measure for exponential Lévy models. Quantitative Finance 6(2), 125-145.

[19] Jacod, J. and Shiryaev, A. N. (2003) Limit theorems for stochastic processes. Berlin: Springer.

[20] Jeanblanc, M., Klöppel, S. and Miyahara, Y. (2007) Minimal $f^{q}$-martingale measures for exponential Lévy processes. Annals of Applied Probability 17, 1615-1638.

[21] Kallsen. J. (2000) Optimal portfolios for exponential Lévy processes. Mathematical Methods of Operations Research 51, 357-374.

[22] Kallsen, J. and Shiryaev, A. N. (2002) The cumulant process and Esscher's change of measure. Finance and Stochastics 6(4), 397-428.

[23] Kohlmann, M. and Xiong, D. (2008) The p-optimal martingale measure when there exist inaccessible jumps. International Journal of Pure and Applied Mathematics 37(3), 321-348.

[24] Kohlmann, M. and Xiong, D. (2008) The minimal entropy and the convergence of the $p$ optimal martingale measures in a general jump model. Stochastic Analysis and Applications 26(5), 941-977.

[25] Krol, K. and Küchler, U. (2009) Minimal entropy martingale measure for Lévy processes. Preprint 9, Institut für Mathematik, Humboldt-Universität zu Berlin (http://www.mathematik.hu-berlin.de/publ/pre/2009/p-list-09.html)

[26] Küchler, U. and Sørensen, M. (1997) Exponential families of stochastic processes. Springer, New York.

[27] Küchler, U. and Tappe, S. (2008) Bilateral Gamma distributions and processes in financial mathematics. Stochastic Processes and their Applications 118(2), 261-283.

[28] Küchler, U. and Tappe, S. (2008) On the shapes of bilateral Gamma densities. Statistics and Probability Letters $\mathbf{7 8}(15), 2478-2484$.

[29] Lewis, A. L. (2001) A simple option formula for general jump-diffusion and other exponential Lévy processes. Envision Financial Systems and OptionCity.net (http://optioncity.net/pubs/ExpLevy.pdf)

[30] Madan, D. B. and Seneta, B. (1990) The VG model for share market returns. Journal of Business 63, 511-524.

[31] Madan, D. B. (2001) Purely discontinuous asset pricing processes. In: Jouini, E., Cvitanič, J. and Musiela, M. (Eds.), pp. 105-153. Option Pricing, Interest Rates and Risk Management. Cambridge University Press, Cambridge.

[32] Mania, M. and Tevzadze, R. (2000) A semimartingale Bellman equation and the varianceoptimal martingale measure. Georgian Mathematical Journal 7(4), 765-792.

[33] Mania, M. and Tevzadze, R. (2003) A semimartingale backward equation and the varianceoptimal martingale measure under general information flow. SIAM Journal on Control and Optimization 42(5), 1703-1726.

[34] Prause, K. (1999) The Generalized Hyperbolic model: estimation, financial derivatives, and risk measures. PhD thesis, University of Freiburg.

[35] Santacroce, M. (2005) On the convergence of the $p$-optimal martingale measures to the minimal entropy martingale measure. Stochastic Analysis and Applications 23(1), 31-54.

[36] Santacroce, M. (2006) Derivatives pricing via $p$-optimal martingale measures: some extreme cases. Journal of Applied Probability 43(3), 634-651.

[37] Sato, K. (1999) Lévy processes and infinitely divisible distributions. Cambridge studies in advanced mathematics, Cambridge. 
[38] Schoutens, W. (2005) Lévy processes in finance. Wiley series in probability and statistics, West Sussex.

[39] Schoutens, W. (2001) The Meixner process in finance. EURANDOM Report 2001-002. EURANDOM, Eindhoven.

[40] Schweizer, M. (1995) On the minimal martingale measure and the Föllmer-Schweizer decomposition. Stochastic Analysis and Applications 13, 573-599.

[41] Schweizer, M. (1996) Approximation pricing and the variance-optimal martingale measure. Annals of Probability 24(1), 206-236.

[42] Sidibé, R. (1979) Martingales locales à accroissements indépendants. In Séminaire de Probabilités XIII, 1977/78, Volume 721 of Lecture Notes in Mathematics, pp. 132-137. Berlin: Springer.

[43] Takuji, A. (2001) The p-optimal martingale measure in continuous trading models. Statistics and Probability Letters 54(1), 93-99.

[44] Takuji, A. (2005) Some properties of the variance-optimal martingale measure for discontinuous semimartingales. Statistics and Probability Letters 74(2), 163-170.

Humboldt University of Berlin, Institute of Mathematics, Unter den Linden 6, D10099 Berlin, Germany; ETH Zürich, Department of Mathematics, Rämistrasse 101, CH8092 ZÜRICH, SWITZERLAND

E-mail address: kuechler@mathematik.hu-berlin.de, stefan.tappe@math.ethz.ch 